\title{
Prevalencia de brucelosis bovina en la cuenca ganadera del Alto Imaza, región Amazonas, Perú
}

\section{Prevalence of Bovine Brucellosis in the Livestock Basin of Alto Imaza, Amazon region, Peru}

\author{
Wan Yú López del Aguila ${ }^{1}$
}

\section{RESUMEN}

El presente estudio tuvo como objetivo evaluar la prevalencia de brucelosis bovina en la cuenca ganadera del Alto Imaza, región Amazonas, Perú. Se trabajó con bovinos de las razas Brown Swiss, y Holstein, sin distinción de edad y sexo, considerando como las razas más representativas en la producción ganadera del Alto Imaza. El estudio se realizó recolectando muestras sanguíneas de los bovinos por venopunción de la vena coccígea ubicada debajo de la cola haciendo uso de tubos al vacío con anticoagulante. Las muestras fueron procesadas mediante el Kit de Rosa de Bengala entre los meses de noviembre 2019 y enero 2020. En los resultados se muestra que según raza; la prevalencia de brucelosis bovina en el estudio demostró que en el 100\% no se evidencia brucelosis, por lo que el número y porcentaje de casos positivos es nulo (0\%). Y según sexo; el estudio también demostró que en el $100 \%$ no se evidencia, por lo que el número y porcentaje de casos positivos es nulo (0\%). Se concluye que la distribución de bovinos del estudio fue: el 32.17\% y $67.83 \%$ fueron de Raza Holstein y Brown Swiss respectivamente. Así mismo el $70 \%$ de los bovinos seleccionados para el estudio fueron vacas en mayor porcentaje, así también el 14\% de tipo vaquilla, el 10\% vaquillonas, y en menor porcentaje con 1,2 y $3 \%$ fueron Toro, Torete y Ternera respectivamente resultando por los distintos tipos y categorías que no existe prevalencia de brucelosis bovina.

\section{Palabras clave: Prevalencia, brucelosis, bovino}

\section{ABSTRACT}

The present study aimed to evaluate the prevalence of bovine brucellosis in the Alto Imaza cattle basin, Amazonas region, Peru. We worked with bovines of the Brown Swiss and Holstein breeds, without distinction of age and sex, considering them as the most representative breeds in the livestock production of Upper Imaza. The study was carried out by collecting blood samples from the bovines by venipuncture of the coccygeal vein located under the tail using vacuum tubes with anticoagulant. The samples were processed using the Rose Bengal Kit between the months of November 2019 and January 2020.The results show that according to race; The prevalence of bovine brucellosis in the study showed that brucellosis is not evidenced in $100 \%$, so the number and percentage of positive cases is zero (0\%). And according to sex; the study also showed that in $100 \%$ there is no evidence, so the number and percentage of positive cases is null ( $0 \%)$. It is concluded that the distribution of cattle in the study was: $32.17 \%$ and $67.83 \%$ were of the Holstein and Brown Swiss breed respectively. Likewise, $70 \%$ of the cattle selected for the study were cows in a higher percentage, as well as $14 \%$ of the heifer type, $10 \%$ heifers, and in a lower percentage with 1, 2 and 3\% were Bull, Torete and Veal respectively resulting from the different types and categories that there is no prevalence of bovine brucellosis.

Keywords: Prevalence, brucellosis, bovine 


\section{INTRODUCCIÓN}

Según la FAO (2019), el concepto de ganadería en el continente americano ha generado gran importancia al ser tenido en cuenta como un producto principal para la canasta básica familiar, el mismo que ayuda a fortalecer la alimentación familiar; también se ha convertido en un sustento económico para muchas familias de los países que forman parte de esta región.

La actividad ganadera en la región del continente americano, según fundamenta la FAO este sector contribuye con el $46 \%$ del PBI, y que en los últimos años ha tenido un incremente significativo anual siendo un $3.7 \%$ más que el aumento en el mundo. Es así que principalmente este sector ha aportado de manera significativa debido al incremento en la demanda de este producto. Con este crecimiento acelerado esta parte de la región del mundo se ha convertido en una de las principales exportadoras del mundo, siendo representativo los productos compuestos por la leche, carne.

Según la FAO en los últimos años los constantes cambios en los espacios en los que se crían y alimentan los animales han producido diversas enfermedades; por lo que la prevención de las mismas ha cobrado relevancia siendo las instituciones las principales embajadoras de este mecanismo con el objetivo de mejorar la producción animal.

En cuanto a la brucelosis bovina para el Instituto Colombiano Agropecuario, esta refiere a una potente mal contagioso al que generalmente se le conoce como aborto infeccioso. Esta enfermedad principalmente afecta a los vacunos en todos los períodos, pero generalmente esta afecta a vacas con gran cantidad de partos; por sobre todo los vacunos de ordeño; también están expuestas a esta enfermedad otro tipo de animales como cerdos, ovejas, cabríos y caballos presentándose en este tipo de animales con diversos síntomas.

Así también para Robles (2012), la brucelosis bovina es definida como una enfermedad que tienen gran capacidad de contagio por su facilidad de contagio, esta generalmente es originada por Brucella abortus. Se caracteriza por afectar a los bovinos recién nacidos por nacer débiles y que al crecer baja la producción de leche, produce infertilidad y reproducción.

Es importante recalcar que la prevención de la enfermedad es la mejor manera de reducir las pérdidas tanto económicas como productivas; uno de las estrategias que ha dado grandes resultados es el cultivo bacteriológico de laboratorio mediante la identificación de las causas.

\section{MATERIAL Y MÉTODO}

Se trabajó con bovinos de las razas Brown Swiss, y Holstein, sin distinción de edad y sexo, considerando como las razas más representativas en la producción ganadera del Alto Imaza.

\section{Tabla 1}

Población de bovinos por distritos según raza.

\begin{tabular}{ccc}
\hline Distrito & \multicolumn{2}{c}{$\begin{array}{c}\text { Población de bovinos por disitos } \\
\text { según raza }\end{array}$} \\
\hline & Brown Swiss & Holstein \\
Granada & 1044 & 26 \\
Olleros & 1816 & 58 \\
Quinjalca & 1051 & 102 \\
\hline
\end{tabular}

Fuente: CENAGRO, 2012

La población estuvo compuesta por un total de 4097 bovinos distribuidos según razas y áreas de estudio datos reportados por CENAGRO, de los cuales se sacó el tamaño de muestra utilizando la fórmula para poblaciones finitas conocidas,

$n=$

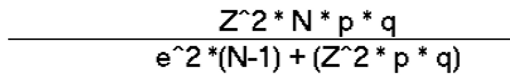

Considerando un nivel de confianza del $90 \%$ y un nivel de error de estimación del $10 \%$ resultando una muestra de 286 bovinos seleccionados mediante el muestreo aleatorio estratificado.

Tabla 2

Muestra de bovinos por distritos según raza.

\begin{tabular}{cccc}
\hline \multirow{2}{*}{ Distrito } & \multicolumn{2}{c}{$\begin{array}{c}\text { Muestra de bovinos por } \\
\text { distritos según raza }\end{array}$} & Total \\
\cline { 2 - 3 } & Brown Swiss & Holstein & \\
\hline Granada & 64 & 19 & 83 \\
Olleros & 66 & 32 & 98 \\
Quinjalca & 64 & 41 & 105 \\
\hline & Total & & 286 \\
\hline
\end{tabular}

El estudio se realizó recolectando las muestras sanguíneas de los bovinos por venopunción de la vena coccígea ubicada debajo de la cola haciendo uso de tubos al vacío con anticoagulante, previa limpieza con alcohol y algodón en el área de punción. Las muestras fueron rotuladas y transportadas bajo refrigeración de $4^{\circ} \mathrm{C}$ al laboratorio del SENASA donde fueron procesadas mediante el Kit de Rosa de Bengala entre los meses de noviembre 2019 y enero 2020 .

Se consideraron los distritos de Granada, Olleros y Quinjalca específicamente en los sectores de Siricha, Olleros, San Miguel, Cashac, Shilmal, Tayahuayco, Chonta, Aymes, Diosan, Calviche, Yerbabuena, Sugmal, perteneciente a la cuenca ganadera del Alto 
Imaza. Para lo cual se trabajó con una muestra de 286 entre vacas, vaquillonas, vaquillas, terneras, toros y toretes distribuidas en razas de Brown Swiss y Holstein.

Resultado positivo: una muestra en que se observó aglutinación en cualquier grado (débilmente perceptible con grumos muy finos hasta aquellas de gruesos grumos, claros y bien definidos).

Resultado negativo: una muestra en la que no se observó aglutinación y la preparación tenía un color rosa uniforme y translúcida al paso de la luz.

\section{RESULTADOS}

Tabla 3

Prevalencia de brucelosis bovina mediante la prueba de rosa de bengala.

\begin{tabular}{lll}
\hline Tipo & Cantidad & Porcentaje \\
\hline Positivo & 0 & $0 \%$ \\
Negativo & 286 & $100 \%$ \\
\hline
\end{tabular}

\section{Tabla 4}

Registro según categoría de los animales, cantidad y resultados de la prueba de Rosa Bengala en la cuenca ganadera del Alto Imaza.

\begin{tabular}{|c|c|c|c|c|c|c|c|c|}
\hline \multicolumn{9}{|c|}{ FICHA DE REGISTRO DE BRUCELOSIS SEGÚN RAZA Y DISTRITO } \\
\hline \multirow[b]{2}{*}{ Distrito } & \multicolumn{8}{|c|}{ CATEGORÍAS DE LOS ANIMALES } \\
\hline & Vaca & Vaquillonas & Vaquilla & Ternera & Toro & Torete & Raza & $\begin{array}{c}\text { Sub } \\
\text { Total }\end{array}$ \\
\hline \multirow{2}{*}{ Olleros } & 20 & 6 & 6 & 0 & 0 & 0 & $\mathrm{H}$ & 32 \\
\hline & 54 & 5 & 7 & 0 & 0 & 0 & B.S. & 66 \\
\hline \multirow{2}{*}{ Quinjalca } & 29 & 7 & 5 & 0 & 0 & 0 & $\mathrm{H}$ & 41 \\
\hline & 49 & 5 & 10 & 0 & 0 & 0 & B.S. & 64 \\
\hline \multirow{3}{*}{ Granada } & 9 & 1 & 5 & 4 & 0 & 0 & $\mathrm{H}$ & 19 \\
\hline & 39 & 5 & 6 & 6 & 2 & 6 & B.S. & 64 \\
\hline & & & Total & & & & & 286 \\
\hline
\end{tabular}

\section{Tabla 5}

Registro según categoría de raza referente a la prevalencia de brucelosis bovina.

\begin{tabular}{ccccccc}
\hline \multicolumn{3}{c}{ FICHA DE REGISTRO DE BRUCELOSIS SEGÚN RAZA } \\
\hline \multicolumn{2}{c}{ Categorías delos Animales Evaluados } & \multicolumn{3}{c}{ Prevalencia de brucelosis } \\
Raza & Cant. & $\%$ & Positivo & \multicolumn{2}{c}{ Negativo } \\
& & & Cant. & $\%$ & Cant. & $\%$ \\
Holstein & 92 & $32.17 \%$ & 0 & $0 \%$ & 92 & $32.17 \%$ \\
Brown Swiss & 194 & $67.83 \%$ & 0 & $0 \%$ & 194 & $67.83 \%$ \\
Total & 286 & $100 \%$ & 0 & $0 \%$ & 286 & $100 \%$ \\
\hline $\begin{array}{c}\text { Brown Swiss } \\
\text { Total }\end{array}$ & 194 & $67.83 \%$ & 0 & $0 \%$ & 194 & $67.83 \%$ \\
\hline
\end{tabular}

\section{Tabla 6}

Registro según categoría de sexo referente a la prevalencia de brucelosis bovina.

\section{FICHA DE REGISTRO DE BRUCELOSIS SEGÚN SEXO}

\begin{tabular}{ccccccc}
\hline Categorías de los Animales Evaluados & \multicolumn{3}{c}{ Prevalencia de brucelosis } \\
& \multicolumn{2}{c}{ Positivo } & \multicolumn{2}{c}{ Negativo } \\
Sexo & Cant. & $\%$ & Cant. & $\%$ & Cant. & $\%$ \\
Macho & 37 & $12.94 \%$ & 0 & $0 \%$ & 37 & $12.94 \%$ \\
Hembra & 249 & $87.06 \%$ & 0 & $0 \%$ & 249 & $87.06 \%$ \\
\hline Total & 286 & $100 \%$ & 0 & $0 \%$ & 286 & $100 \%$ \\
\hline
\end{tabular}


Tabla 5

Registro según categoría de raza referente a la prevalencia de brucelosis bovina.

\begin{tabular}{cccc}
\hline \multicolumn{4}{c}{$\mid$ POBLACIÓN DE BOVINOS POR DISTRITOS SEGÚN RAZA } \\
\hline DISTRITO & BROWN SWISS & HOLSTEIN & TOTAL \\
Granada & 1044 & 26 & 1070 \\
Olleros & 1816 & 58 & 1874 \\
Quinjalca & 1051 & 102 & 1153 \\
\hline Total & 3911 & 186 & 4097 \\
\hline
\end{tabular}

\section{Tabla 8}

Muestra de bovinos por distritos según raza

\begin{tabular}{cccccccc}
\hline \multicolumn{8}{c}{ Muestra de bovinos por distritos según raza } \\
\hline \multicolumn{7}{c}{ Brown Swiss } & \multicolumn{2}{c}{ Holstein } & \multicolumn{3}{c}{ Total } \\
Distrito & Cant. & \% & Cant. & \% & Cant. & \% \\
Granada & 64 & 22.38 & 19 & 6.64 & 83 & 29.02 \\
Olleros & 66 & 23.08 & 32 & 11.19 & 98 & 34.27 \\
Quinjalca & 64 & 22.38 & 41 & 14.34 & 105 & 36.71 \\
Total & 194 & 67.83 & 92 & 32.17 & 286 & $100 \%$ \\
\hline
\end{tabular}

\section{DISCUSIÓN}

Zambrano y Pérez (2015), en su artículo titulado, seroprevalencia de brucelosis en ganado bovino y en humanos vinculados a la ganadería bovina en las zonas norte y centro de la provincia Manabí, Ecuador. Llegó a los siguientes resultados teniendo en cuenta que la investigación también se realizó con la técnica de Rosa de Bengala como prueba tamiz; tal y como se desarrolló en la investigación materia de discusión: con el estudio se demostró que la provincia Manabí presenta una baja prevalencia de animales y de humanos afectados y una alta prevalencia de hatos afectados. En tal sentido la investigación desarrollada muestra resultados totalmente diferentes que en su $100 \%$ reveló que todos los hatos materia de estudio no muestran prevalencia de brucelosis; es decir no se evidencia un diagnostico positivo en el estudio, el mismo que favorece para seguir proyectando planes que prevengan y fortalezcan el cuidado para evitar dicha enfermedad.

Maslucán (2018), con su investigación, diagnóstico de la prevalencia de Brucelosis bovina en los hatos ganaderos mediante la prueba serológica (Rosa de bengala) en el distrito de Pardo Miguel - Naranjos. Llegó a los siguientes resultados: La prevalencia de la brucelosis bovina según las variables sexo, raza, etapa productiva y habitad en el distrito de Pardo Miguel es de $0 \%$, es así que el porcentaje de los hatos ganaderos expuestos a brucelosis bovina es de $0 \%$ ya que no se encontró ninguna muestra positiva a la prueba serológica rosa de bengala, demostrando que los hatos ganaderos del distrito de pardo miguel se encuentra libre de la infección bacteriana. Teniendo en cuenta los resultados de la presente investigación se manifiesta la existencia de resultados análogos, ya que en ambos estudios realizados no se evidenció prueba alguna de la existencia de la enfermedad correspondiente a brucelosis; por lo tanto, es un lugar seguro para el consumo humano principalmente de la leche que se manifiesta como un conducto para la transportación de dicha enfermedad.

Según Bardales (2017), con su investigación prevalencia de brucelosis bovina en las cuencas Mashcón y Chonta - Cajamarca, 2016; llegó a la siguiente conclusión: La prevalencia de brucelosis bovina utilizando prueba de Rosa bengala, basado en la justificación de positivos y prueba de fijación de complemento es de $0.13 \%$ o $13 \%$ de efectividad. Al respecto la presente investigación muestra un resultado con mayor efectividad que la investigación citada anteriormente; pues en el estudio se evaluó con la misma prueba, pero con la prueba $\mathrm{Z}$ de proporciones encontrando diferencias proporcionales toda vez que el $\mathrm{Z}$ calculado es de 1.55 una cantidad menor que el $Z$ crítico de \pm 1.96 , lo que permitió aseverar y a la vez aceptar la hipótesis nula confirmando con un nivel de significancia del 95\% que no hay prevalencia de brucelosis bovina en la cuenca ganadera del Alto Imaza. Y teniendo en cuenta el porcentaje de efectividad con el estudio se demostró un $55 \%$ de efectividad con $42 \%$ mayor que el 13\% demostrado en el estudio de Bardales.

\section{CONCLUSIONES}

Según la prueba $\mathrm{Z}$ de proporciones tenemos que el $\mathrm{Z}$ calculado es de 1.55 una cantidad menor que el $\mathrm{Z}$ crítico de \pm 1.96 , lo que nos permite aseverar y a la vez aceptar la hipótesis nula confirmando con un nivel de significancia del $95 \%$ que no hay prevalencia de brucelosis bovina en la cuenca ganadera del Alto 
Imaza.

La prevalencia de brucelosis bovina en el estudio demostró que en el $100 \%$ no se evidencia, por lo que el número y porcentaje de casos positivos es nulo $(0 \%)$. Y según sexo; el estudio también demostró que en el $100 \%$ no se evidencia, por lo que el número y porcentaje de casos positivos es nulo $(0 \%)$.

La distribución de bovinos del estudio fue: el 32.17\% y $67.83 \%$ fueron de Raza Holstein y Brown Swiss respectivamente. Así mismo el 70\% de los bovinos seleccionados para el estudio fueron vacas en mayor porcentaje, así también el $14 \%$ de tipo vaquilla, el $10 \%$ vaquillonas, y en menor porcentaje con 1,2 y $3 \%$ fueron Toro, Torete y Ternera respectivamente resultando por los distintos tipos y categorías que no existe prevalencia de brucelosis bovina.

\section{REFERENCIAS BIBLIOGRÁFICAS}

Bardales, M. (2017). Prevalencia de brucelosis bovina en las Cuencas Mashcón y Chonta Cajamarca, 2016. (Tesis para Título). Universidad Nacional de Cajamarca, Cajamarca. Recuperado el 12 de Agosto de 2020, de http://repositorio.unc.edu.pe/ bitstream/handle/UNC/1135/Tesis\%20Mig uel $\% 20$ Bardales.pdf? sequence $=1 \&$ is Allow $\mathrm{ed}=\mathrm{y}$

FAO. (10 de Agosto de 1991). Directrices para reforzar los servicios de sanidad animal en los países en desarrollo. Obtenido de http://www.fao.org/3/u2200s/u2200s02.ht $\mathrm{m}$

FAO. (23 de Noviembre de 2019). FAO: La ganadería y sus desafios en América Latina y el Caribe. Obtenido de Agronews Castilla y Leon: https://www.agronewscastillay leon.com/fao-la-ganaderia-y-sus-desafiosen-america-latina-y-el-caribe

Maslucán, J. (2018). Diagnóstico de la prevalencia de Brucelosis bovina en los hatos ganaderos mediante la prueba serológica (Rosa de bengala) en el distrito de Pardo Miguel Naranjos. (Tesis de Título). Universidad Nacional de San Martin, Tarapoto. Recuperado el 12 de Agosto de 2019, de http://repositorio.unsm.edu.pe/bitstream/ha ndle/11458/3268/MED.\%20VETERINARI A \%20-\%20Jagner\%20Masluc\%C3\%A $1 \mathrm{n} \% 20 \mathrm{Golac}$.pdf? sequence $=1 \&$ is Allowed $=\mathrm{y}$
Robles, C. (2012). Brucelosis bovina. Patagonia: Grupo de Salud Animal - INTA Bariloche. Recuperado el 03 de enero de 2020, de https://inta.gob.ar/sites/default/files/scripttmp-inta_ganaderia04_brucelosis_bovin a.pdf

Zambrano, M. y. (2015). Seroprevalencia de brucelosis en ganado bovino y en humanos vinculados a la ganadería bovina en las zonas norte y centro de la provincia Manabí, Ecuador. Scielo, 1-8. Recuperado el 12 de Agosto de 2019, de http://scielo.sld.cu/ scielo.php? script $=$ sci arttext\&pid $=$ S0253570X2015000300004 